Supporting Information

\title{
SElF-Organization OF Microscale Condensate FOR Delayed FLOODING OF NANOSTRUCTURED SUPERHYDROPHOBIC SURFACES
}

\author{
Emre Ölçeroğlu and Matthew McCarthy* \\ Department of Mechanical Engineering and Mechanics, \\ Drexel University, 3141 Chestnut St, Philadelphia PA 19104, USA \\ *Corresponding author: mccarthy@coe.drexel.edu
}




\section{S1. Video Files}

\section{Supporting Video \#1}

Optical microscopy imaging of condensation on superhydrophobic and mixed-wettability $(\mathrm{P}=25 \mu \mathrm{m})$ surfaces at $\Delta \mathrm{T}=5^{\circ} \mathrm{C}$.

Supporting Video \#2

ESEM imaging of droplet self-organization at a supersaturation of $S=1.54$.

\section{Supporting Video \#3}

ESEM imaging of condensation on superhydrophobic and mixed-wettability $(\mathrm{P}=25 \mu \mathrm{m})$ surfaces at a supersaturation of $S=1.06$, showing increased nucleation site density.

Supporting Video \#4

ESEM imaging of condensation on superhydrophobic and mixed-wettability $(\mathrm{P}=25 \mu \mathrm{m})$ surfaces at a supersaturation of $S=1.54$, showing delayed flooding.

\section{Supporting Video \#5}

Optical imaging of condensation on superhydrophobic surfaces from $\mathrm{N}_{2}$ with $100 \%$ relative humidity at atmospheric pressure for various saturation temperature differences, showing a transition to a flooded state prior to $\Delta \mathrm{T}=20^{\circ} \mathrm{C}$. The formation of a liquid film is observable at the bottom portion of the $\Delta \mathrm{T}=20{ }^{\circ} \mathrm{C}$ panel, indicating flooding. This film slowly progresses up the chip resulting in complete failure and flooding of the surface.

Supporting Video \#6

Optical imaging of condensation on mixed-wettability surfaces $(P=25 \mu \mathrm{m})$ from $\mathrm{N}_{2}$ with $100 \%$ relative humidity at atmospheric pressure for various saturation temperature differences, showing no surface flooding up to $\Delta \mathrm{T}=25^{\circ} \mathrm{C}$ and a transition from jumping to continuous shedding. 


\section{S2. Virus-Templated Nanostructured Surfaces}

The Tobacco mosaic virus (TMV) is a benign cylindrical plant virus, which has an $18 \mathrm{~nm}$ outer diameter and $300 \mathrm{~nm}$ length. ${ }^{1,2}$ The TMV used in this work is the TMV1cys, an engineered mutant of the wild-type virus containing a cysteine residue on the outer surface of its coat proteins. ${ }^{3}$ This results in enhanced binding properties, allowing for near-vertical assembly and metallization onto various substrates, creating highly textured three-dimensional metal-coated nanostructures. ${ }^{4}$ The TMV solutions used in this study are prepared through the inoculation of tobacco plants (Nicotiana tabacum cultivar Xanthi). When seedlings are 3-4 weeks old they are infected with the TMV by rubbing existing solutions onto the leaves. The infected tobacco grows for 2-3 weeks, and TMV replicates during the infection process. The leaves are collected and stored at $-20^{\circ} \mathrm{C}$ until the purification process begins. The TMV is extracted by blending the plant material using a standard kitchen blender. A series of chemical and centrifuging steps are then used to purify and separate the TMV from the rest of the plant material. The purified TMV is finally diluted to $0.1 \mathrm{~g} / \mathrm{L}$ using $0.1 \mathrm{M}$ sodium phosphate buffer. The self-assembly and metallization of surface-bound TMV is explained in Experimental Section in main paper.

(a) TMV Biotemplating Process

(b)

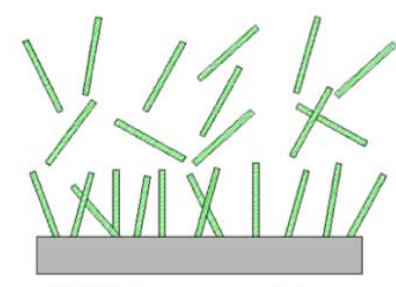

TMV assembly

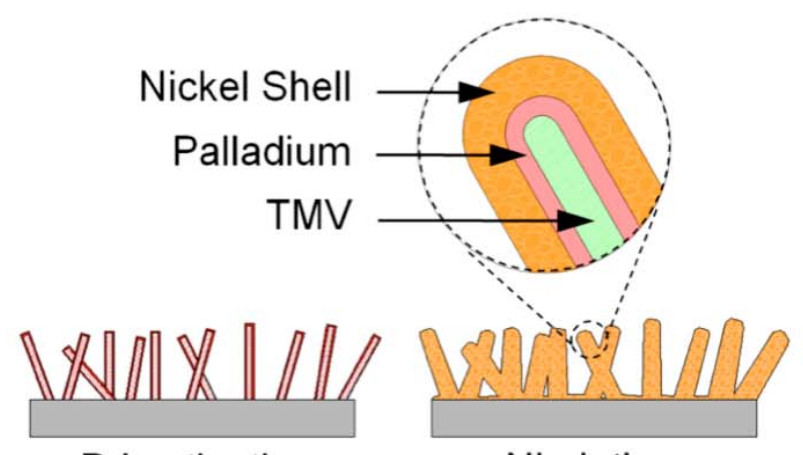

Pd activation

Ni plating
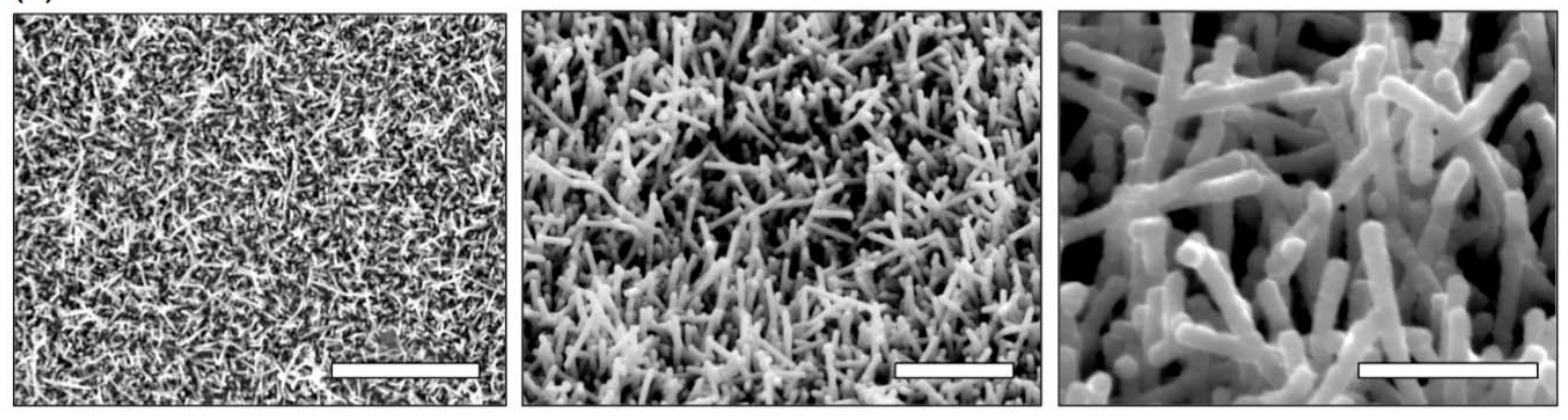

Figure S1. Biotemplated nanofabrication using the Tobacco mosaic virus (TMV) showing (a) the three-step solution-based room-temperature process and (b) SEM imaging of the resulting nanostructured coating at various magnifications. Scale bars are $5 \mu \mathrm{m}, 2 \mu \mathrm{m}$, and $1 \mu \mathrm{m}$. 


\section{S3. Experimental Characterization}

\section{S3.1 Optical Microscopy Apparatus}

Figure S2 shows a schematic representation of the custom-built apparatus used for characterization during optical microscopy. Samples were cleaned with IPA, rinsed with DI water, dried with $\mathrm{N}_{2}$, and dehydrated at $100{ }^{\circ} \mathrm{C}$ for 10 minutes. The samples are then fixed to the cold plate using thermal grease to minimize thermal resistance. Dry $\mathrm{N}_{2}$ was constantly pumped into the chamber to bring the enclosure RH down to $\sim 1 \%$. When the chamber reached dry condition, the sample temperature was set to the desired value by adjusting the constant temperature bath. A three-way valve was then used to switch from dry $\mathrm{N}_{2}$ to humid $\mathrm{N}_{2}$ sparged through a bath of water at ambient temperature to initiate condensation. During condensation, the vapor properties and surface temperature are measured while the high-speed camera collects images of the resulting droplet nucleation, growth, and removal. More details about this set-up is explained in previous work. ${ }^{5}$

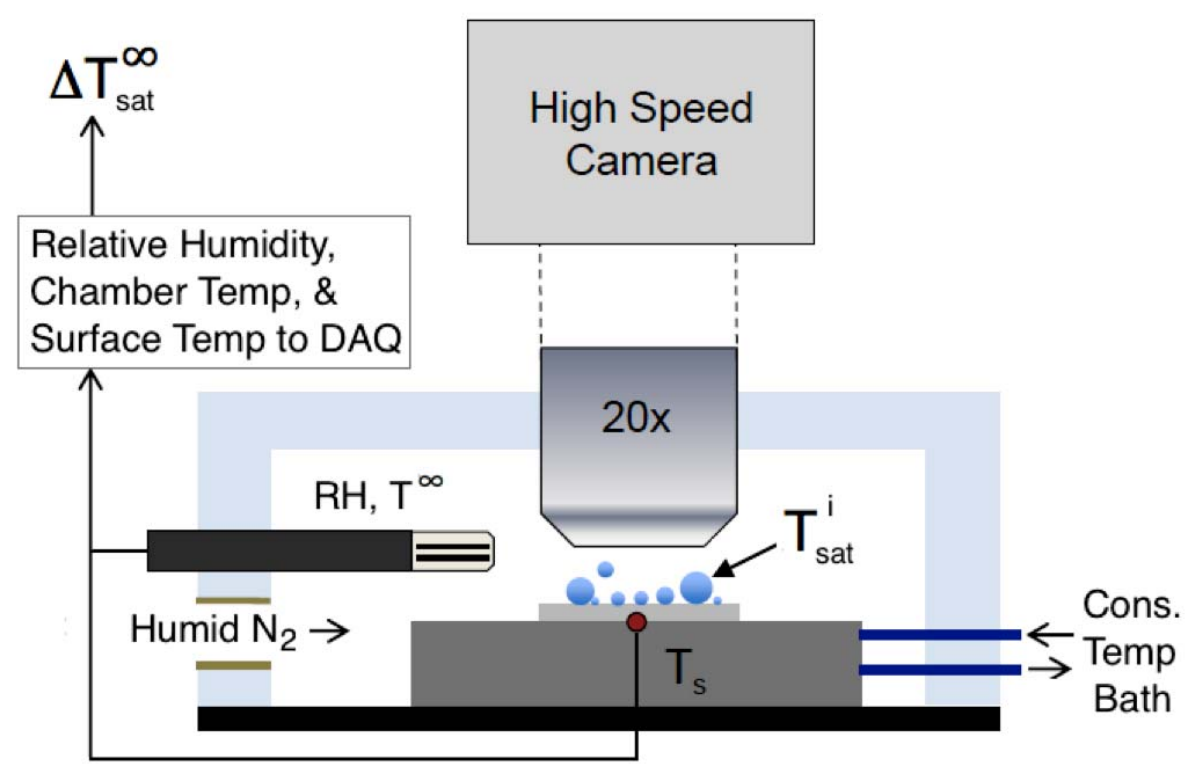

Figure S2. Schematic of the optical microscopy experimental apparatus used for characterization of condensation from humid $\mathrm{N}_{2}$ in a controlled environment.

\section{S3.2 Environmental Chamber}

Figure S3 shows a schematic representation of the system used to characterize condensation at large supersaturations from humid air. An environmental chamber was used to test a larger range of sub-cooling conditions $\left(5^{\circ} \mathrm{C}<\Delta T<25^{\circ} \mathrm{C}\right)$ than can be achieved in the optical microscopy set-up shown in Figure S2. The sample is mounted vertically and exposed to $\mathrm{N}_{2}$ at $100 \%$ relative humidity while the cooling block controls the sample sub-cooling. The high-speed camera is coupled with Infinity KC long-distance microscope and IF3 microscope objective lens to provide longer working distances. Using this set-up imaging through extremely humid conditions $(\approx 100 \% \mathrm{RH})$ was possible. 


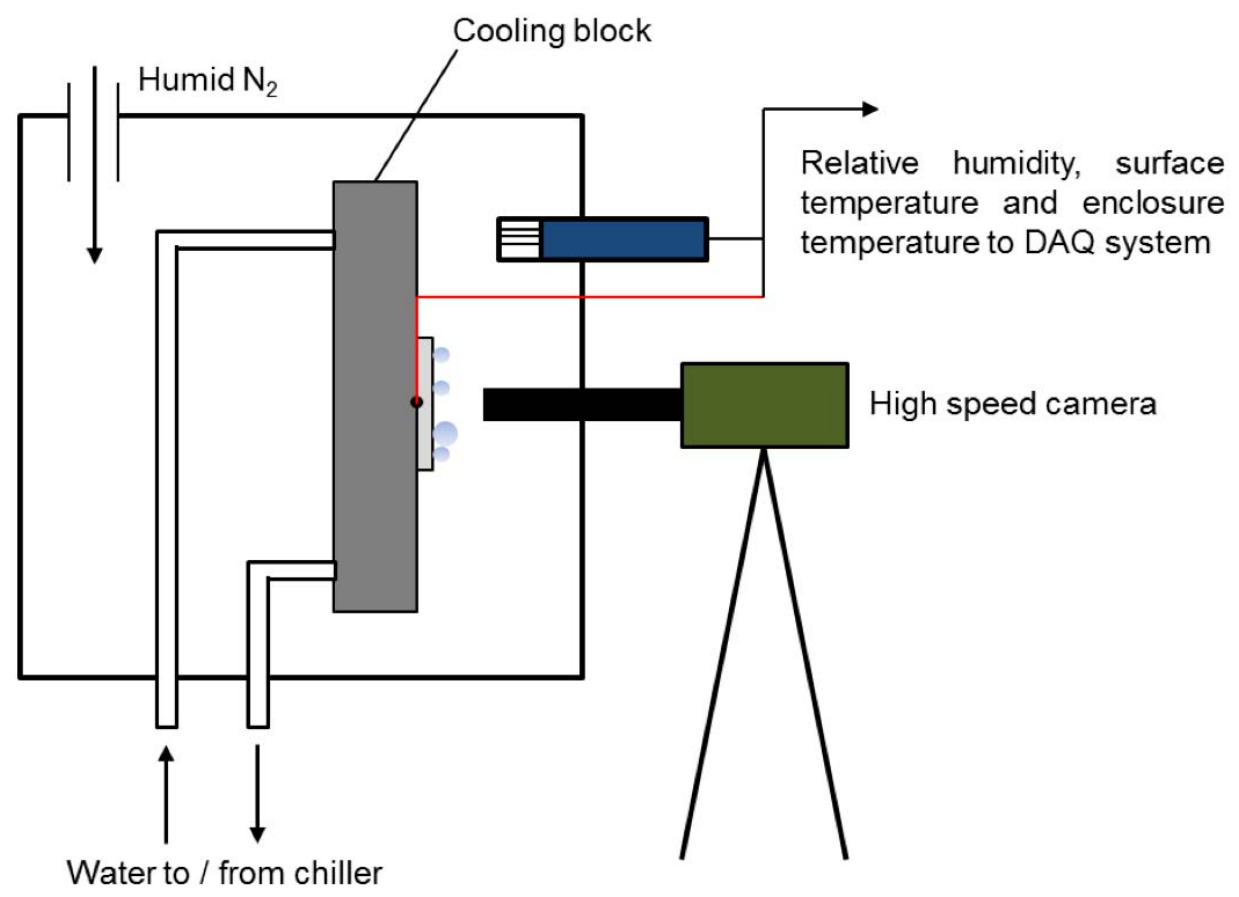

Figure S3. Schematic of the custom-built environmental chamber used to image condensation from humid $\mathrm{N}_{2}$ over a range of sub-cooling conditions.

\section{S4. Optical Microscopy at Low Supersaturations}

Six different mixed-wettability designs were characterized using optical microscopy. While the nucleation site diameter was held constant at $\mathrm{d}=3 \mu \mathrm{m}$ for each design, the pitch $(\mathrm{P})$ was varied from 10 to $40 \mu \mathrm{m}$. Figure $\mathrm{S} 4$ shows time-lapse images from the characterization of each MW surface tested in the manner described in the manuscript. The transient response of each surface ramped quickly to a sub-cooling of $5{ }^{\circ} \mathrm{C}$ is shown.

Each frame of the complete videos (captured at $24 \mathrm{fps}$ ) is individually processed using a custombuilt image-tracking algorithm using MATLAB to measure the position and size of each droplet. $^{5}$ Figure S5 shows image processing results characterizing the ability of the surfaces to promote spatial order as well as the resulting surface coverage. Figure S5a shows the percentage of droplets with diameters less than the engineered pitch $(\mathrm{D}<\mathrm{P})$ that are located on the engineered sites. For surfaces with $\mathrm{P} \leq 25 \mu \mathrm{m}$, it is seen that $>90 \%$ of the droplets are located on the engineered sites. Figure S5b shows the transient surface coverage of select MW designs as compared to superhydrophobic surfaces. For all designs with $\mathrm{P} \geq 20 \mu \mathrm{m}$, a steady state condition was reached with droplets continually ejecting. For $\mathrm{P} \leq 15$ no ejections were observed; the droplets continued to coalesce and grow on the surface. 

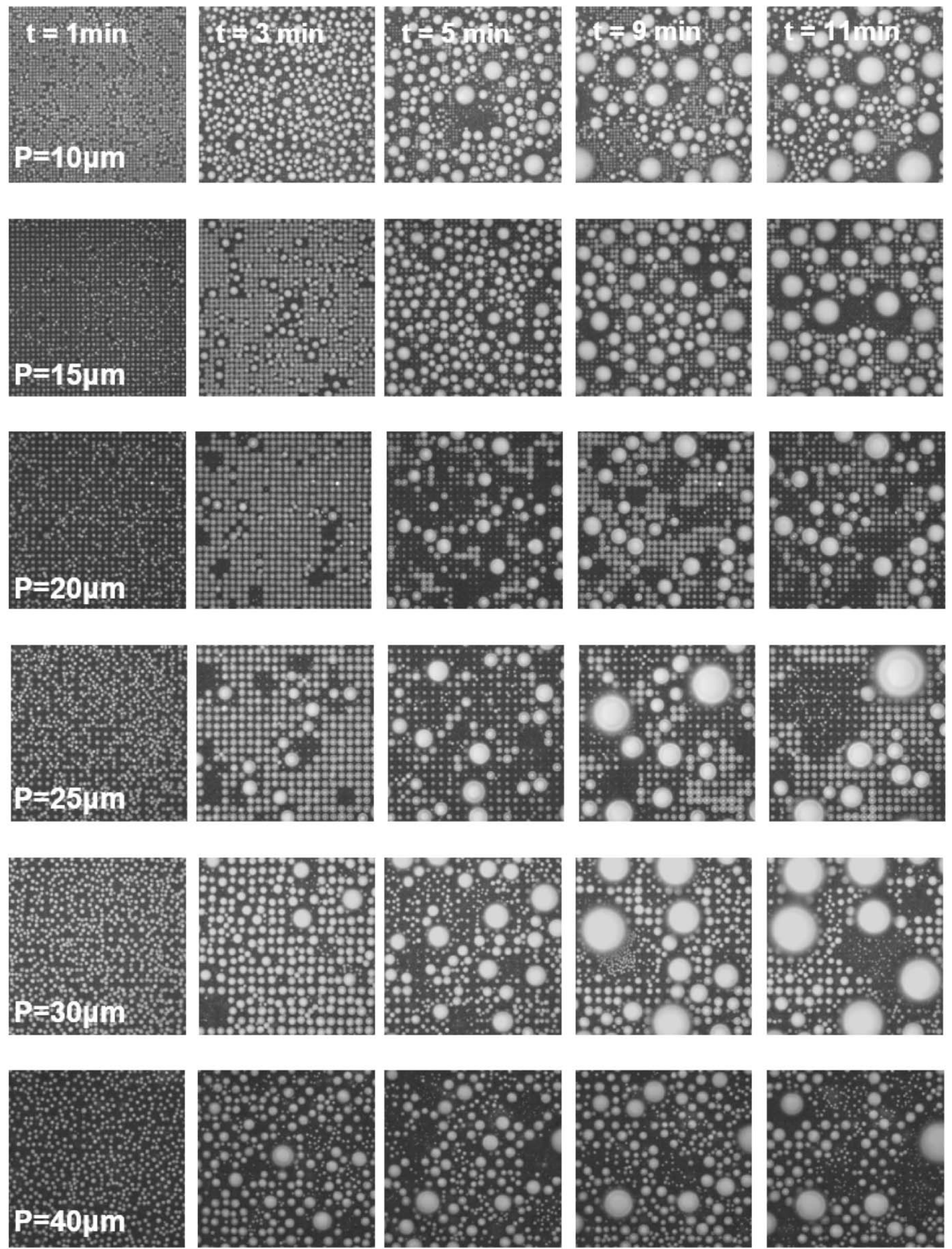

Figure S4. Time-lapse images of condensation on horizontally oriented mixed-wettability surfaces $\left(\Delta T=5^{\circ} \mathrm{C}\right)$ characterized using optical microscopy. 

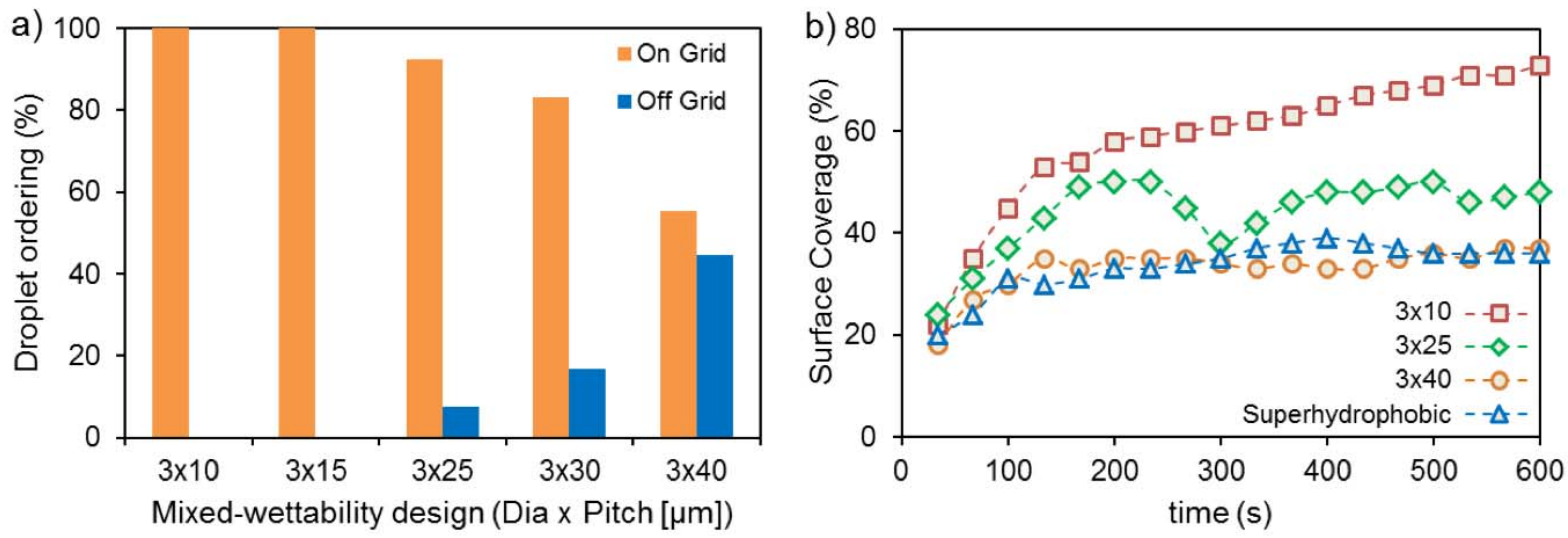

Figure S5. Image processing results from optical microscopy, showing (a) the percentage of small droplets $(\mathrm{D}<\mathrm{P})$ growing on the engineered sites for each design, and (b) the transient surface coverage of each surface during condensation after an abrupt increase in sub-cooling.

\section{S5. Critical Energy Analysis}

The efficiency of the droplet jumping phenomena has been studied previously by Enright et al. using numerical techniques. ${ }^{6}$ Using a $2 \mathrm{D}$ axisymmetric simulation, and accounting for the effects of surface adhesion and viscous dissipation, they reported that the droplet jumping velocity follows an inertial-capillary scaling of the form

$$
V_{\text {Jump }}=\Phi \sqrt{\frac{2 \gamma}{\rho D}}
$$

where $\gamma$ and $\rho$ are the surface tension and density of the liquid, and $D$ is the diameter of two identical droplets immediately prior to coalescence. A series of simulations were conducted for two-droplet events with varying, but equal, droplet diameters. It was found that the proportionality constant, $\Phi$, was a function of the Ohnesorge number

$$
O h=\frac{4 \mu}{\sqrt{\rho \gamma D}}
$$

where $\mu$ is the droplet viscosity. Curve fitting of the numerical results yielded a quadratic dependence of

$$
\Phi=f(O h)=C_{1} O h^{2}+C_{2} O h+C_{3}
$$

where $\mathrm{C}_{1}=3.4026, \mathrm{C}_{2}=-1.5285, \mathrm{C}_{3}=0.2831$. This allowed for the determination of the jumping efficiency defined as the ratio of the kinetic energy of the resultant jumping droplet, $E_{\text {Kinetic }}$, relative to the total excess surface energy available, $\Delta E$, 


$$
\eta_{\text {Jump }}=\frac{E_{\text {Kinetic }}}{\Delta E}=\frac{\Phi^{2}}{3\left(N-N^{2 / 3}\right)}
$$

where $N$ indicates the number of coalescing droplets. This approach was used to show that the jumping efficiency of microscale droplets varies anywhere between $1 \%$ and $5 \%$.

Equation S4 was derived for the coalesecne of two perfectly spherical droplets with no surface contact, thus neglecting the the energy required to overcome adhesion or cohesion. This showed that only a small percentage (1-5\%) of the excess surface energy was actually available for conversion into kinetic energy. By assuming that all of the energy available to be converted into kinetic energy was instead used to overcome the work of cohesion $\left(E_{\text {Kinetic }}=W_{C o h}\right)$, then a critical energy to overcome surface cohesion can be defined. Combining Equation S2 through Equation S4 and setting the droplet diameter equal to the droplet diameter at coalesence on a MW surface $(\mathrm{D}=\mathrm{P})$ yields

$$
\left(\frac{W_{\text {Coh }}}{\Delta E}\right)_{\text {Critical }}=\frac{1}{3\left(N-N^{2 / 3}\right)}\left[\left(\frac{16 C_{1} \mu^{2}}{\rho \gamma}\right) P^{-1}+\left(\frac{4 C_{2} \mu}{\sqrt{\rho \gamma}}\right) P^{-1 / 2}+C_{3}\right]^{2}
$$

Equation S5 represents the maximum energy available to overcome cohesive forces for the coalescing of two droplets on a MW surfaces. It should be noted that Equation S3 was determined for two-droplet coalesence events, and therefore Equation S5 is only applicable for the $\mathrm{N}=2$ case.

\section{S6. References}

1. H. Fraenkel-Conrat, R. C. Williams, Reconstitution of Active Tobacco Mosaic Virus From its Inactive Protein and Nucleic Acid Components, Proc. Nat. Acad. Sci. USA 1955, 41, 690.

2. R. D. Leapman, N. W. Rizzo, Towards Single Atom Analysis of Biological Structures, Ultramicroscopy 1999, 78, 251.

3. S.-Y. Lee, J. Choi, E. Royston, D. B. Janes, J. N. Culver, M. T. Harris, Deposition of Platinum Clusters on Surface-Modified Tobacco Mosaic Virus, J. Nanosci. Nanotechnol. 2006, 6, 974.

4. E. Royston, A. Ghosh, P. Kofinas, M. T. Harris, J. N. Culver, Self-Assembly of VirusStructured High Surface Area Nanomaterials and Their Application as Battery Electrodes, Langmuir 2007, 24, 906.

5. E. Ölçeroğlu, C.-Y. Hsieh, M. M. Rahman, K. K. S. Lau, M. McCarthy, Full-Field Dynamic Characterization of Superhydrophobic Condensation on Biotemplated Nanostructured Surfaces, Langmuir 2014, 30, 7556.

6. R. Enright, N. Miljkovic, J. Sprittles, K. Nolan, R. Mitchell, E. N. Wang, How Coalescing Droplets Jump, ACS Nano 2014, 8, 10352. 